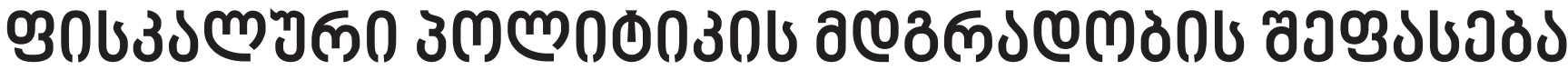

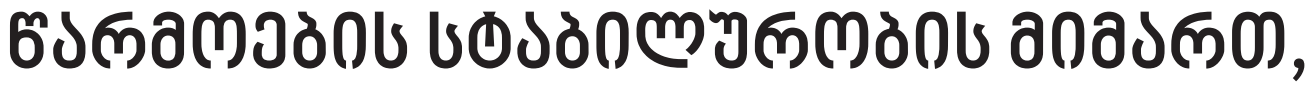

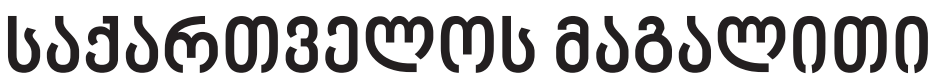

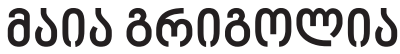

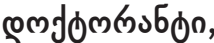

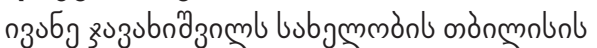

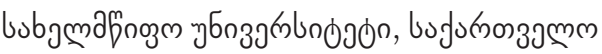

m.grigolia@iset.ge

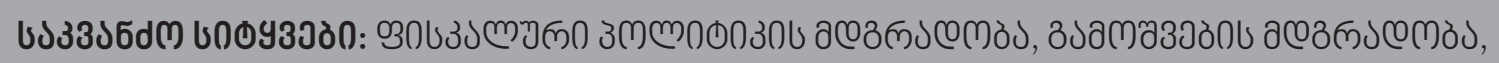

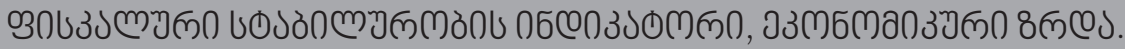

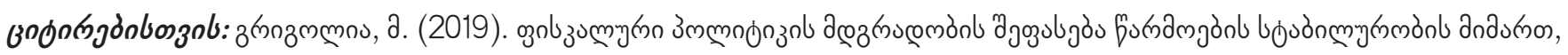

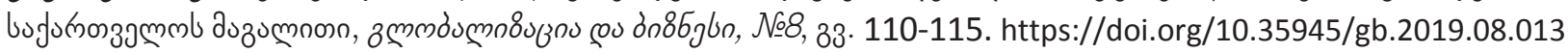

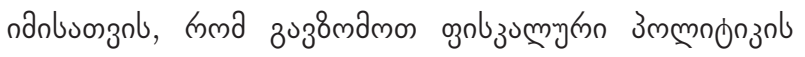

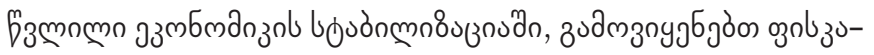

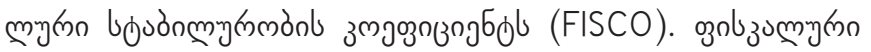

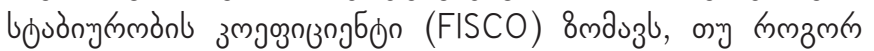

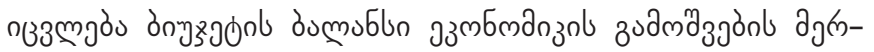

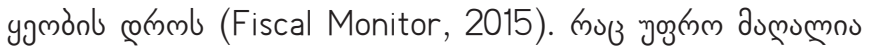

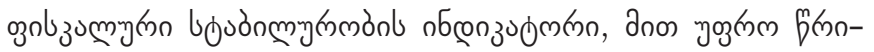

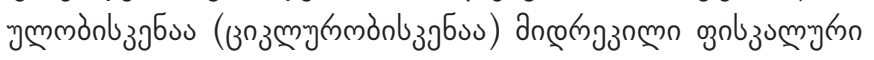

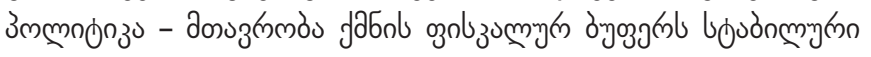

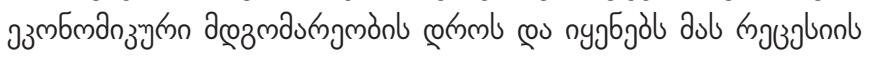

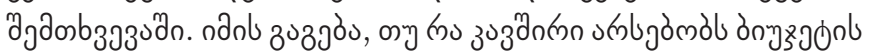

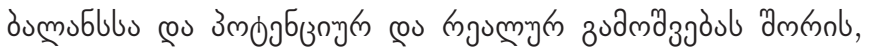

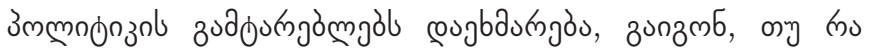

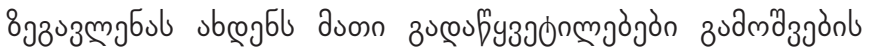

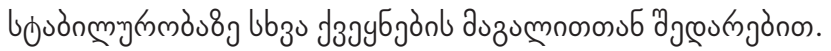

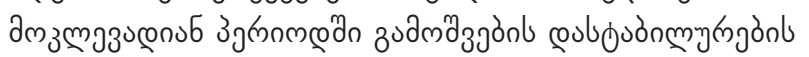

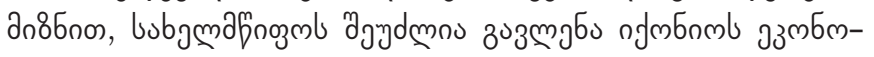

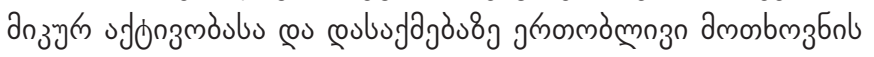

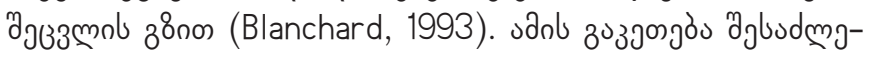

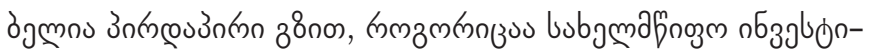

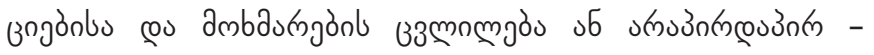

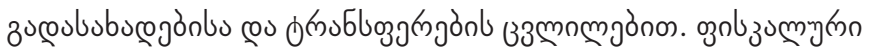

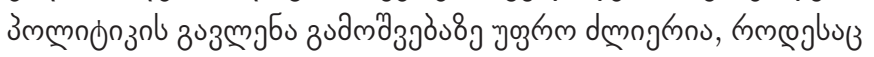

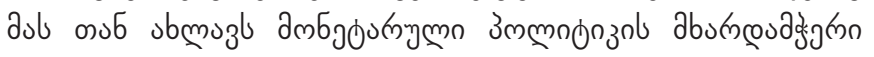

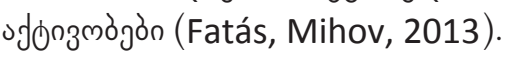

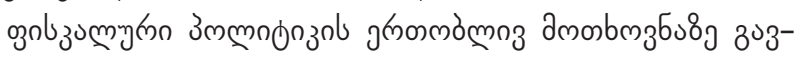

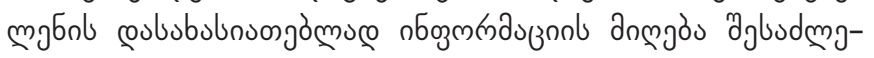

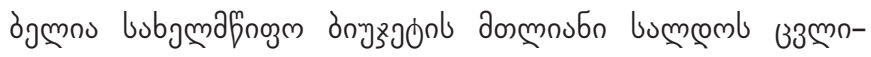

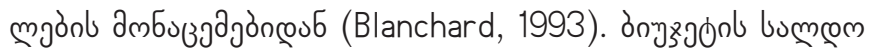

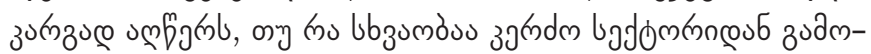

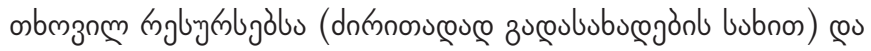

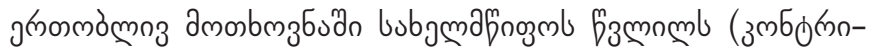

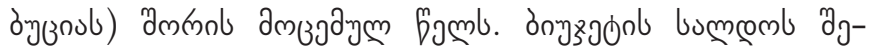

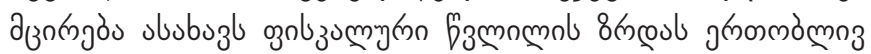
amosbm 36 бon (Fund, 2015).

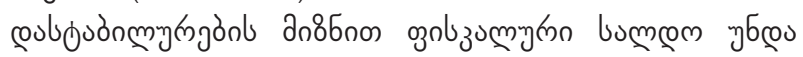

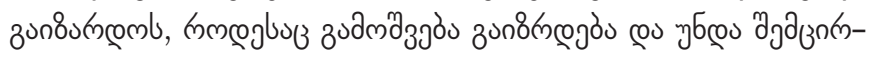

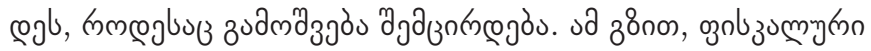

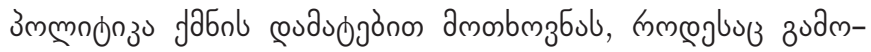

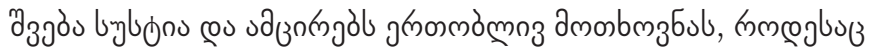

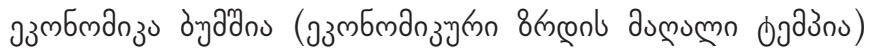
(Girouard, Andre, 2005). sanomadus gुnlzu

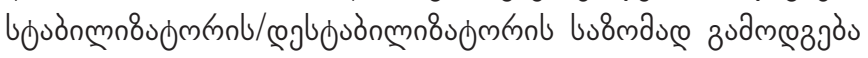

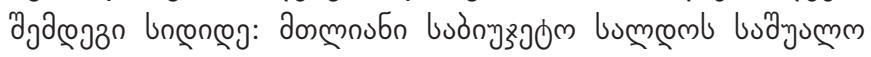

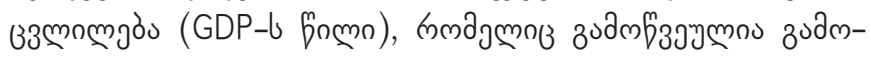

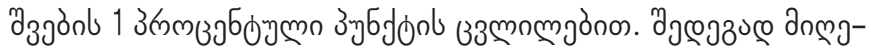

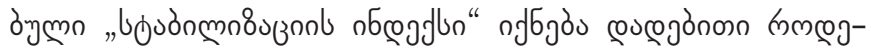

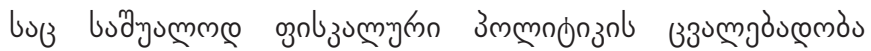

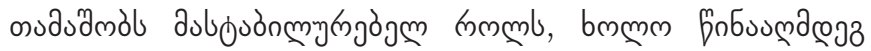

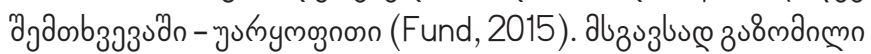

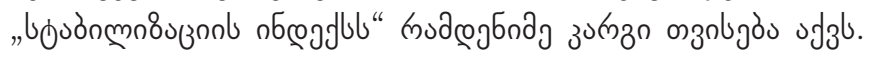

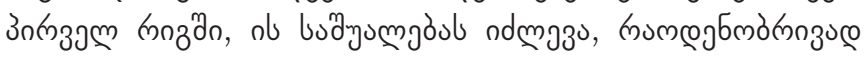

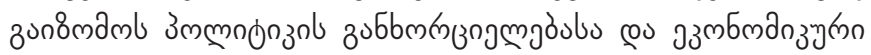

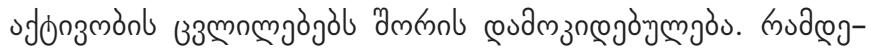




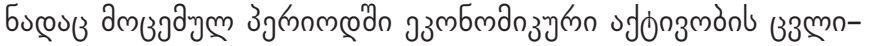

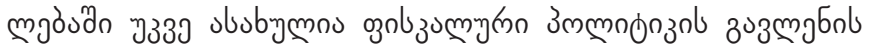

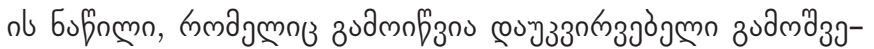

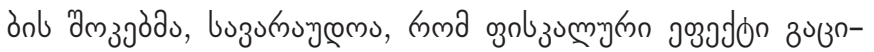

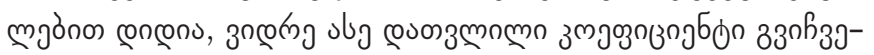

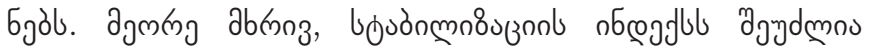

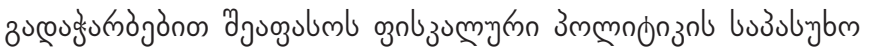

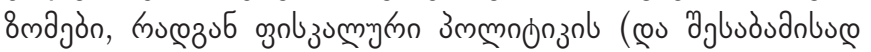

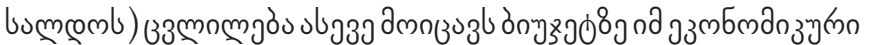

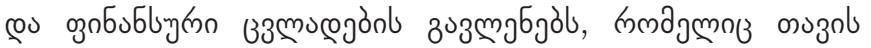

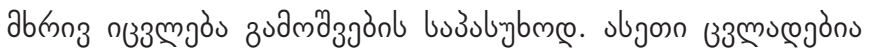

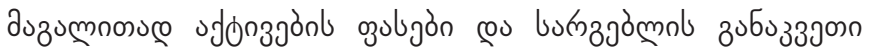
(Bénétrix, Lane, 2013).

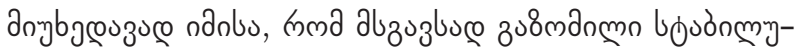

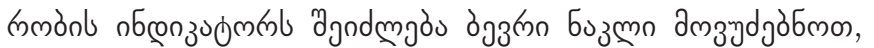

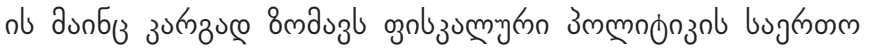

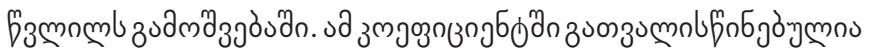

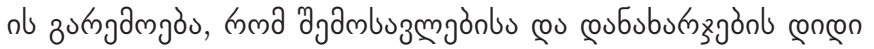

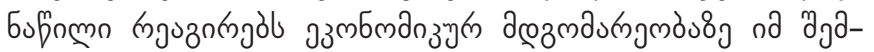

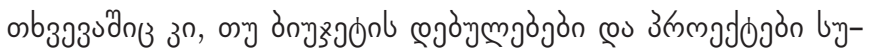

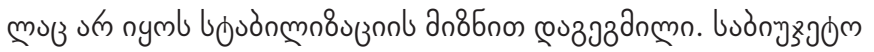

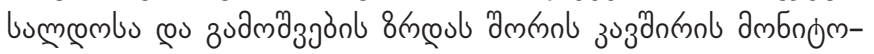

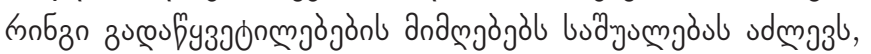

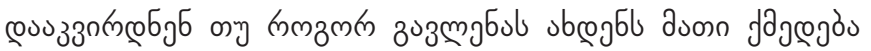

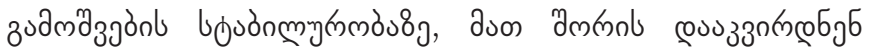

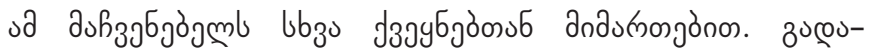

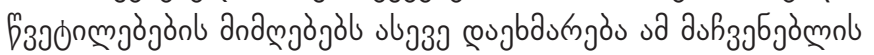

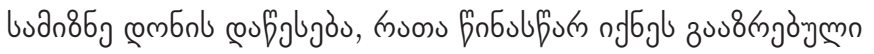

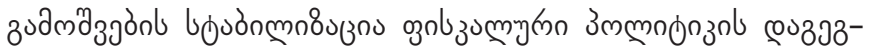

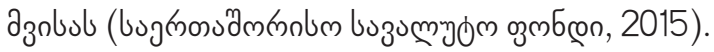

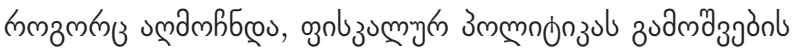

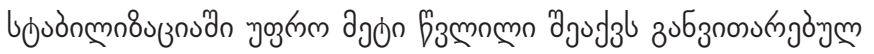

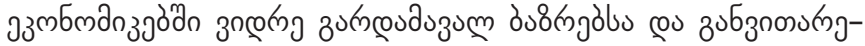

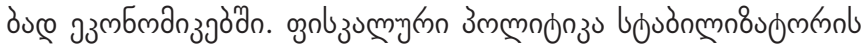

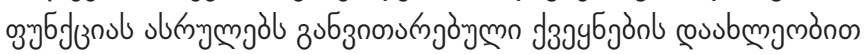

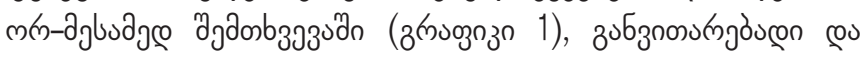

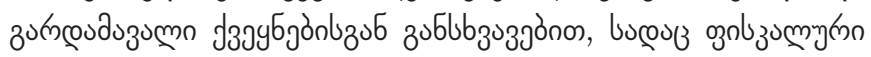

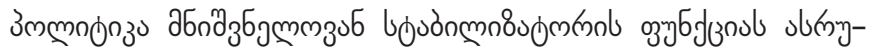

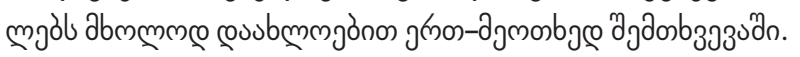

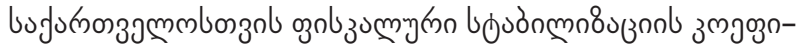

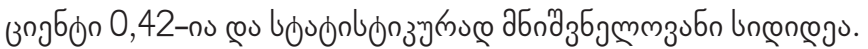

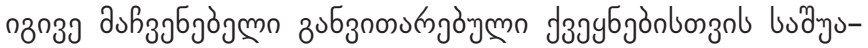

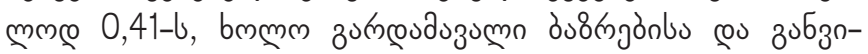

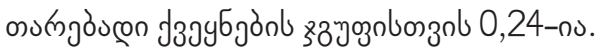

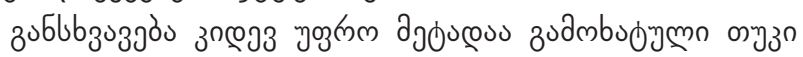

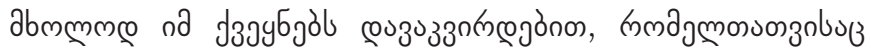

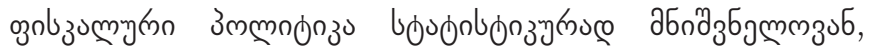

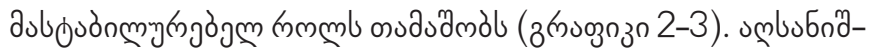

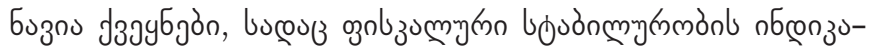

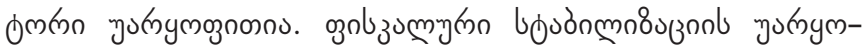

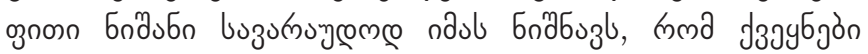

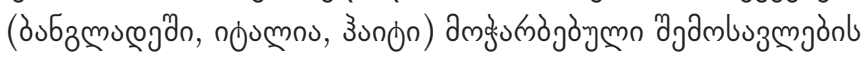

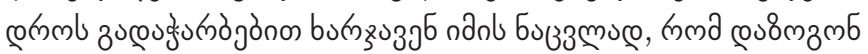

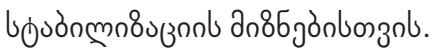

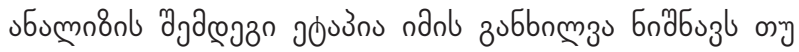

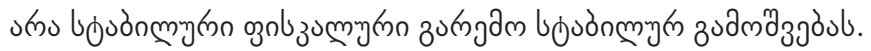

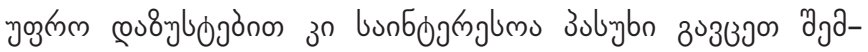

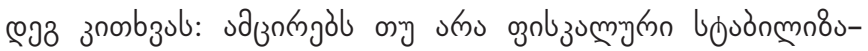

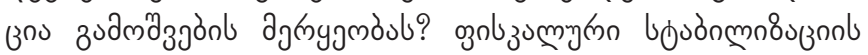

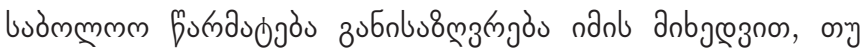

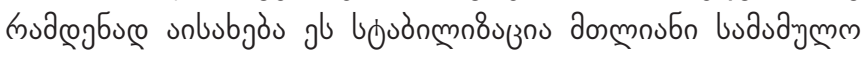

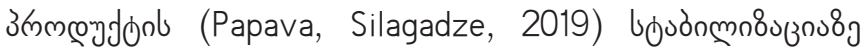

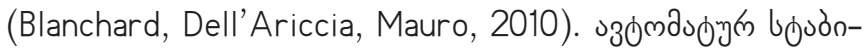

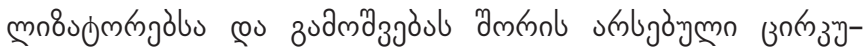

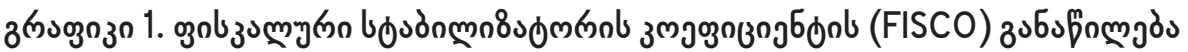

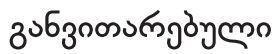

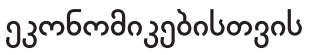

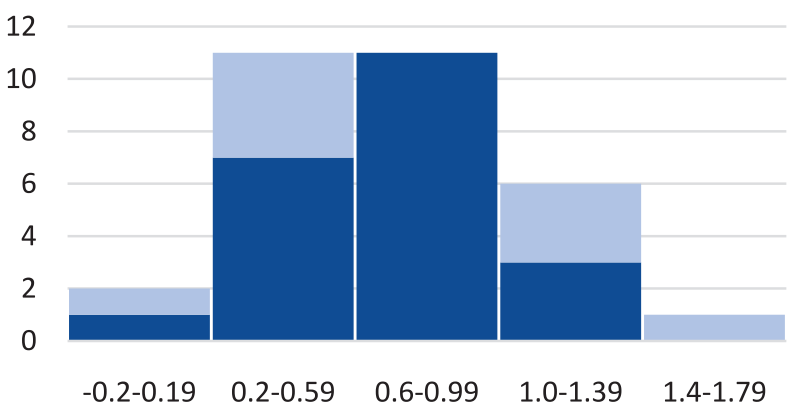

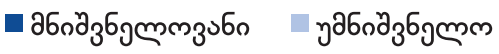
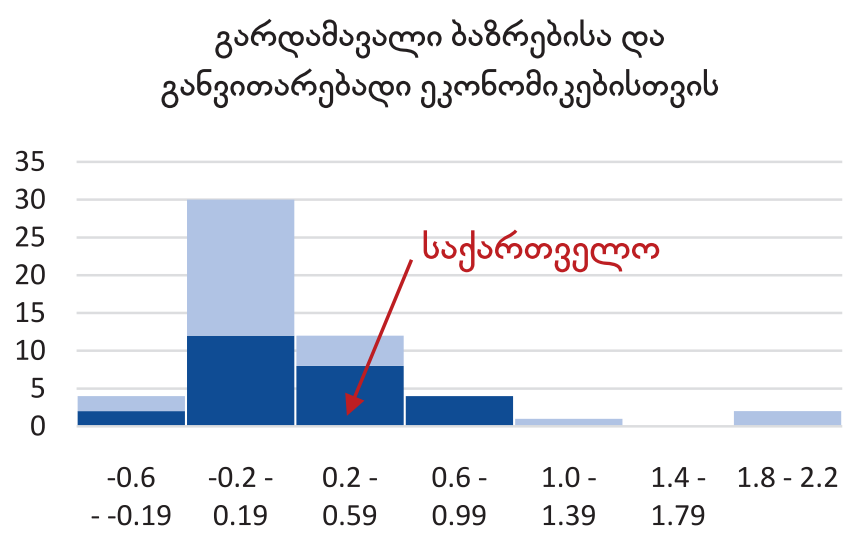

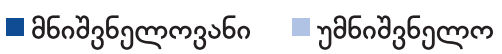

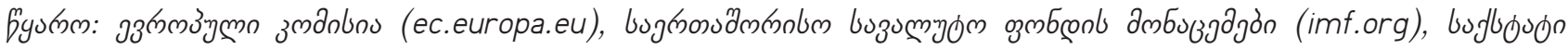

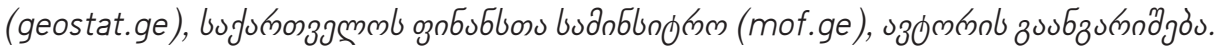




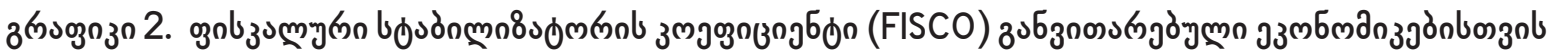

1,5

1

0,5

0

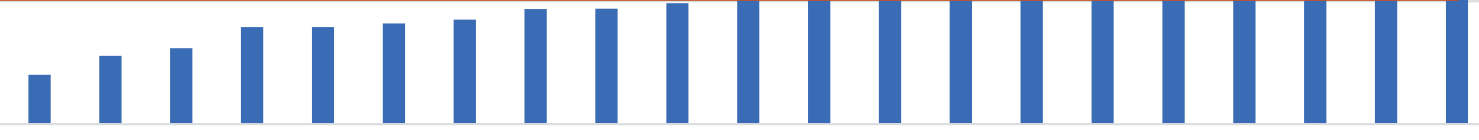

$-0,5^{0}$ ปै

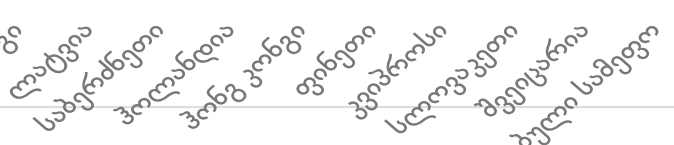

$-1$
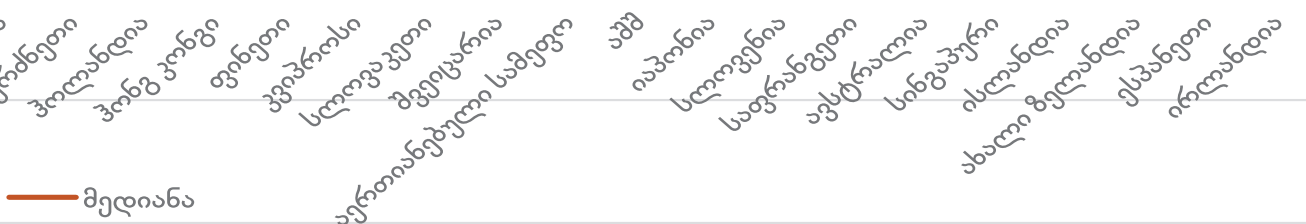

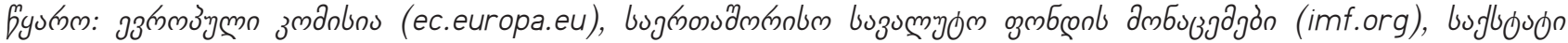

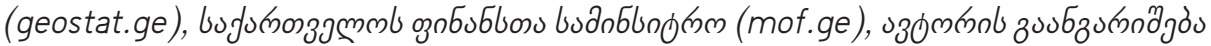

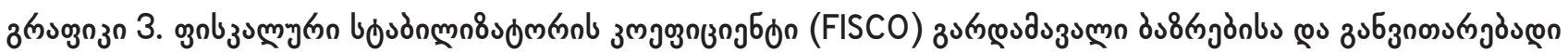

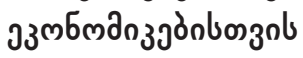

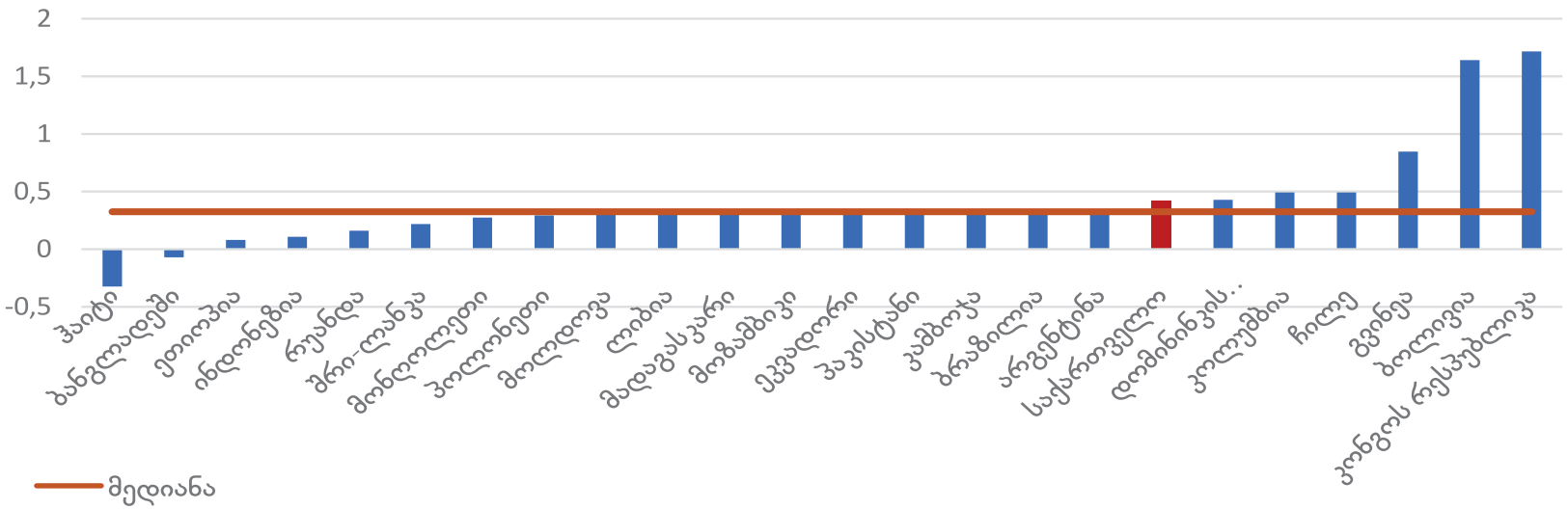

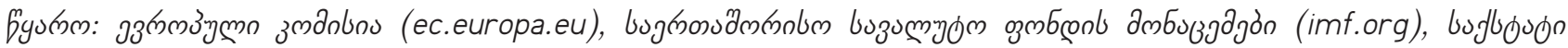

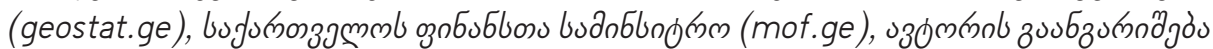

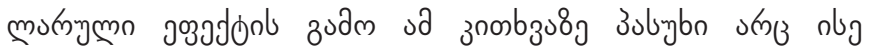

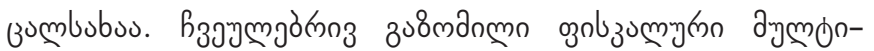

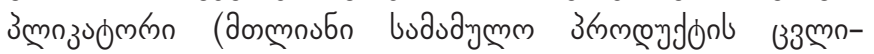

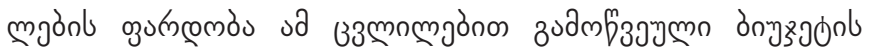

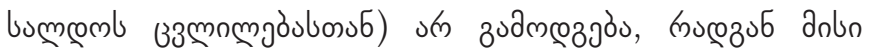

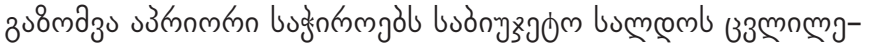

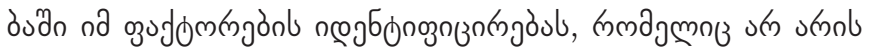

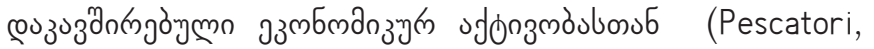

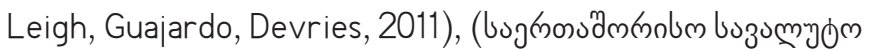
gुmb(on, 2012).

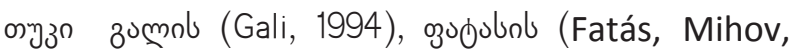

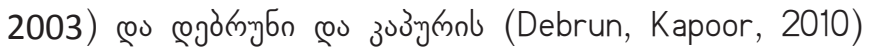

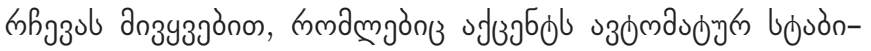

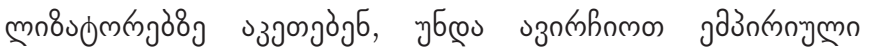

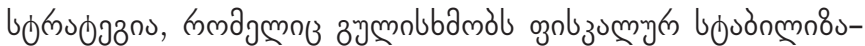

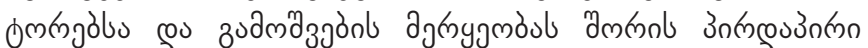

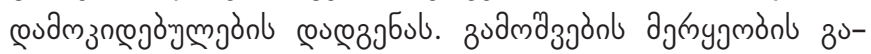

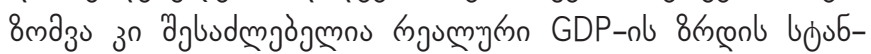

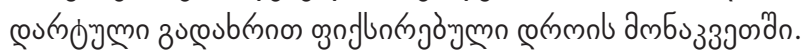

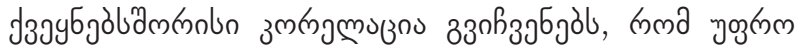

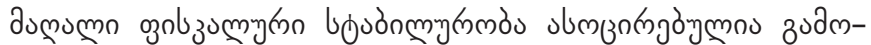

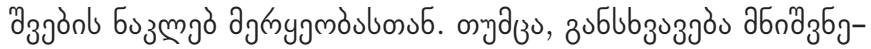

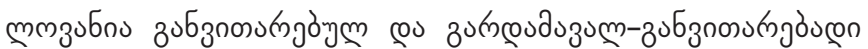

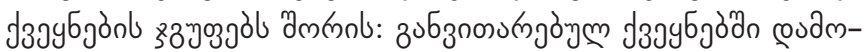

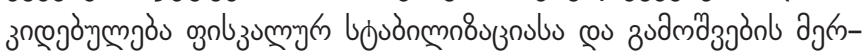

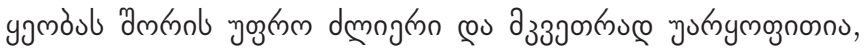

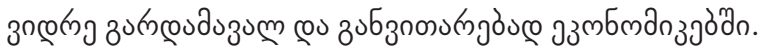

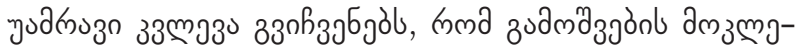




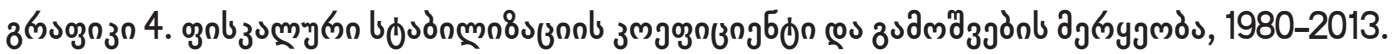
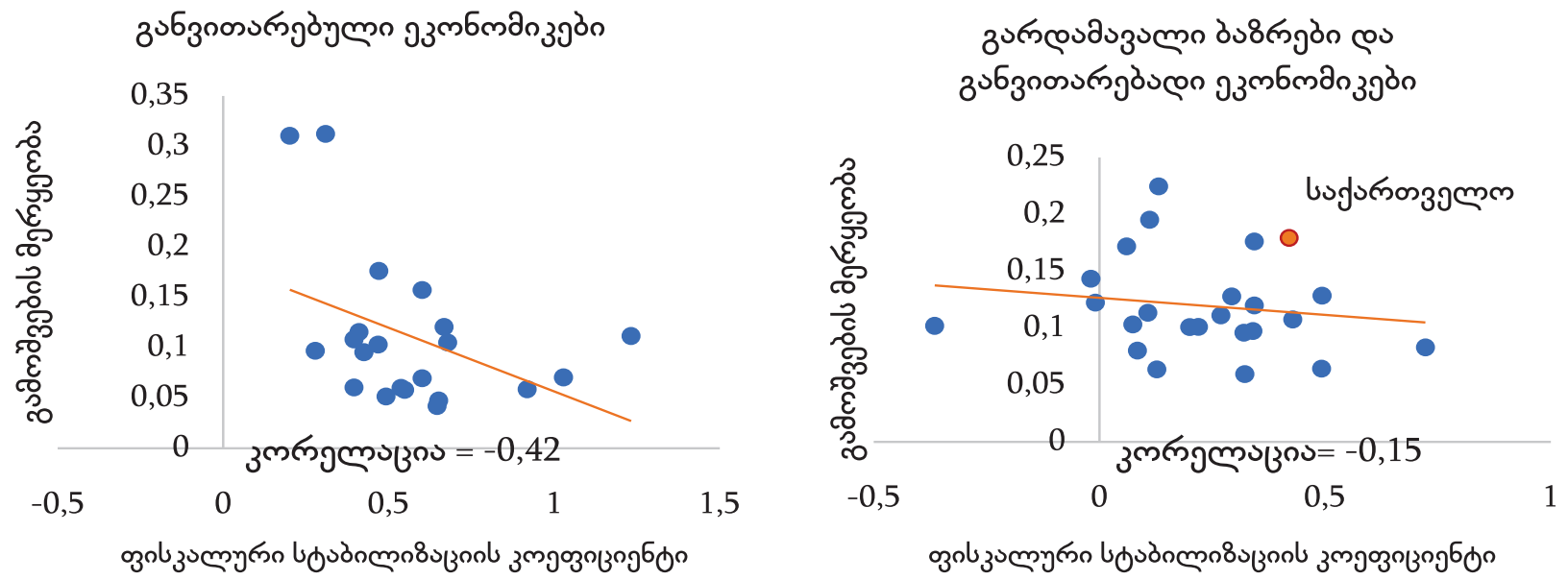

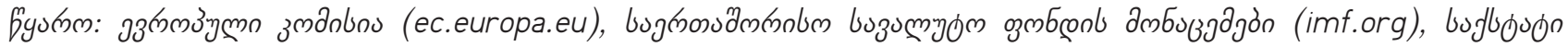

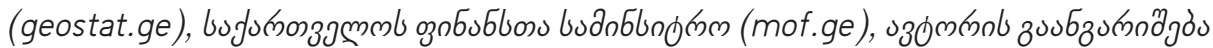

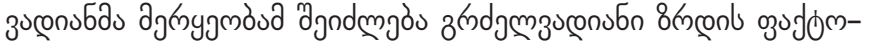

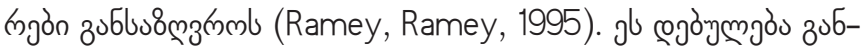

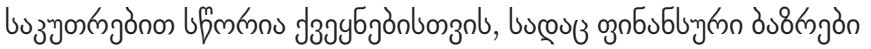

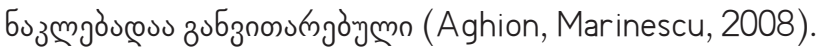

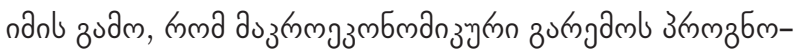

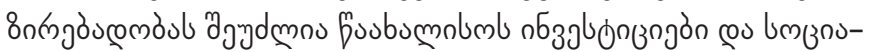

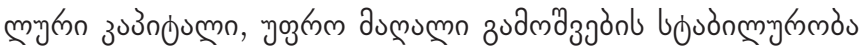

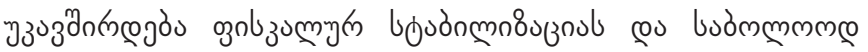
एuмgjonn

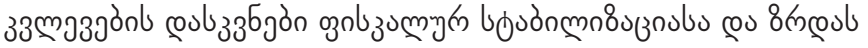

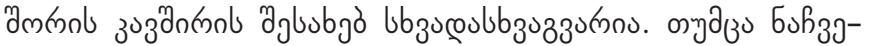

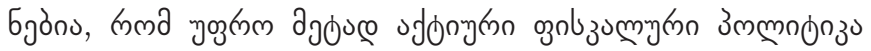

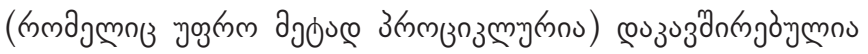

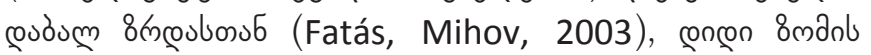

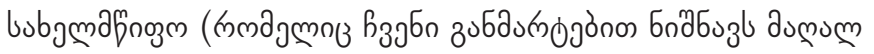

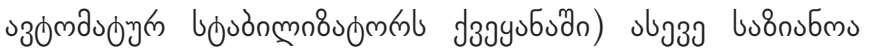
8 menonbonzol (Afonso, Furceri., 2010), (Afonso, 2012).

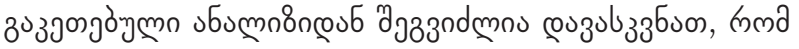
zuдm

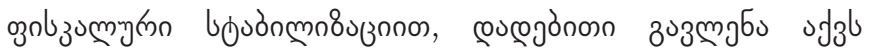

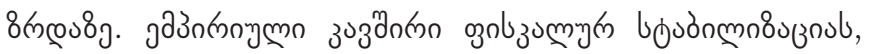

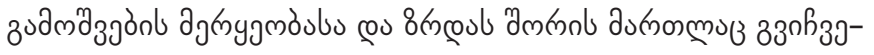

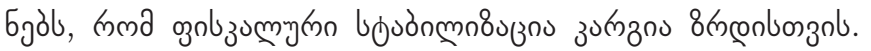

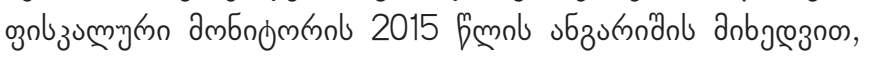

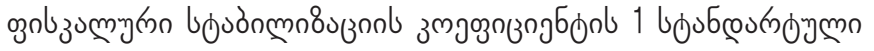

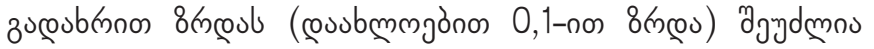

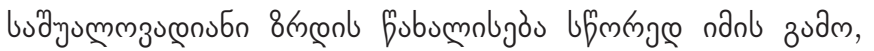

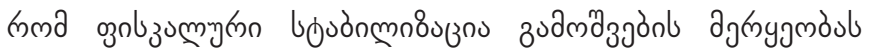

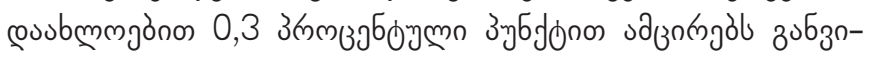

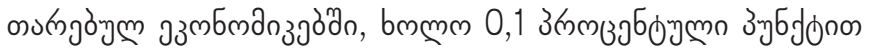

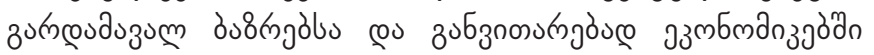
(Fiscal Monitor, 2015).

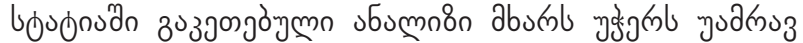

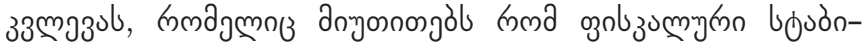

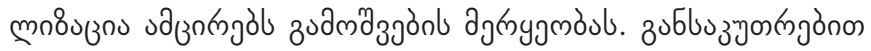

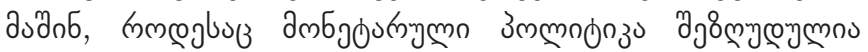

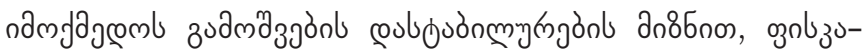

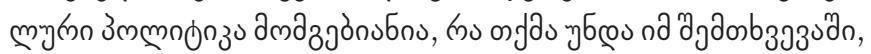

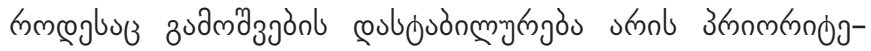

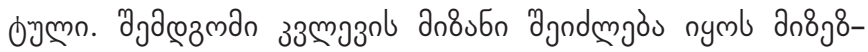

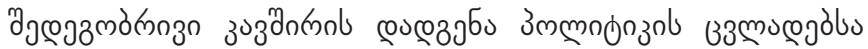

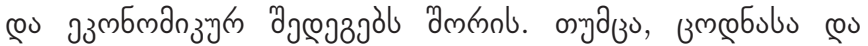

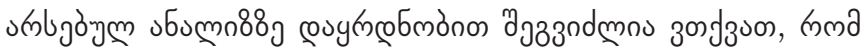

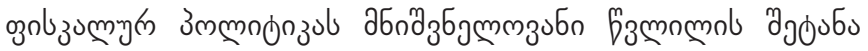

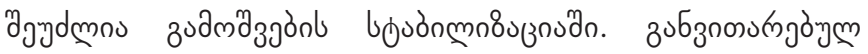

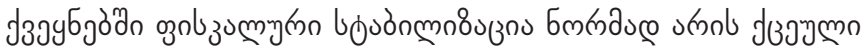

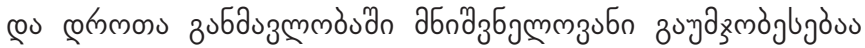

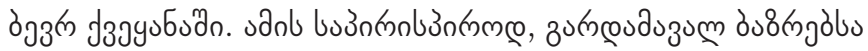

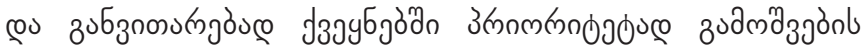

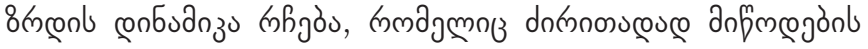

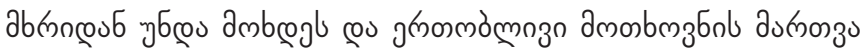

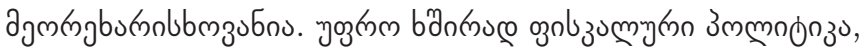

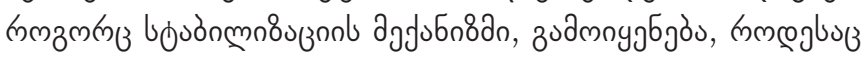

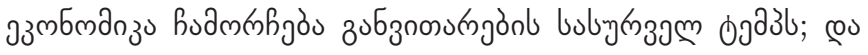

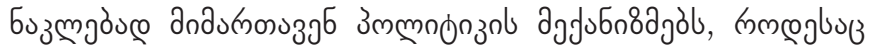

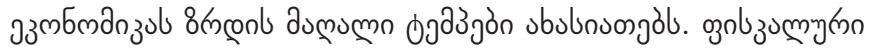

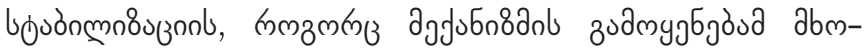

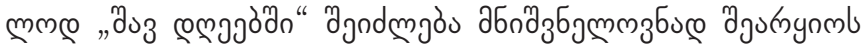

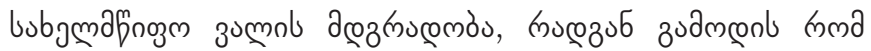

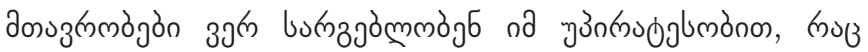

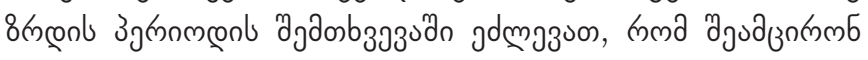

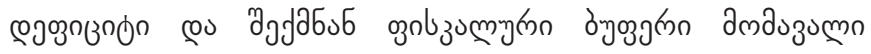

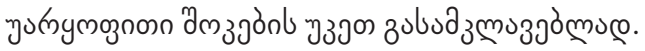




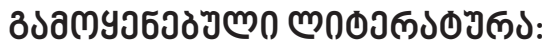

1. Papava, V., Silagadze, A. (2019, April), On the Georgian name of one key economic term "Gross Domestic Product" [Ertiani sakvandzo terminis "Gross Domestic Product"-is qartuli sakheltsodebis shesaxkheb]. Economics and Business, pp. 180-182. (In Georgian).

2. International Monetary Fund (2012, April) Fiscal Monitor. Balancing Fiscal Policy Risks.

3. International Monetary Fund (2015, April) Fiscal Monitor. Now is the time:Fiscal Policy for Sustainable Growth.

4. International Monetary Fund (2018, April) Fiscal Monitor: Capitalizing on Good Times.

5. Afonso, A. J. (2012). Fiscal Volatility, Financial Crisis and Growth. Applied Economics Letters, 1821-26.

6. Afonso, A., and D. Furceri. (2010). Government Size, Composition, Volatility and Economic Growth. European Journal of, 517-32.

7. Aghion, P., Marinescu, I. (2008). Cyclical Budgetary Policy and Economic Growth: What Do We Learn from OECD Panel Data? NBER Macroeconomics Annual, Vol 22.

8. Baunsgaard, T., Symansky, A. (2009). Automatic Fiscal Stabilizers. International Monetary Fund, IMF Staff Position Note $09 / 23$.

9. Bénétrix, A. S., Lane P. R. (2013). Fiscal Cyclicality and EMU. Journal of International Money and Finance, 164-76.

10. Blanchard, O. J. (1993). Suggestions for a New Set of Fiscal Indicators. In H. A. Verbon, The Political Economy of Government Debt. Elsevier Science.

11. Blanchard, O. J., Dell'Ariccia, G., Mauro, P. (2010). Rethinking Macro Policy. International Monetary Fund, IMF Staff Position Note 10/03.

12. Debrun, X., Kapoor, R. (2010). Fiscal Policy and Macroeconomic Stability: New Evidence and Policy Implications. Nordic Economic Policy Review, 35-70.

13. Fatás, A. Mihov, I. (2013). Policy Volatility, Institutions and Economic Growth. Review of Economics and Statistics, 362-76.

14. Fatás, A., Mihov, I. (2001). Government Size and Automatic Stabilizers: International and Intranational Evidence. Journal of International Economics, 3-28.

15. Fatás, A., Mihov, I. (2003). The Case for Restricting Fiscal Policy Discretion. Quarterly Journal of Economics, 1419-47.

16. Fund, I. M. (2015, April). Fiscal Monitor. Now Is the Time-Fiscal Policies for Sustainable Growth.

17. Gali, J. (1994). Government Size and Macroeconomic Stability. European Economic Review, 117-32.

18. Girouard, N., Andre, C. (2005). Measuring Cyclically-Adjusted Budget Balances for OECD Countries. OECD.

19. Pescatori, A., Leigh, D., Guajardo, J., Devries, P. (2011). A New Action-based Dataset of Fiscal Consolidation. International Monetary Fund, Working Paper 11/128.

20. Ramey, G., Ramey, M. (1995). Cross-Country Evidence on the Link between Volatility and Growth. American Economic, 1138-51.

21. www.geostat.ge

22. www.mof.ge

23. www.imf.org

24. www.ec.europa.eu

25. www.OECD.org 


\title{
OUTPUT VOLATILITY IMPACTS ON FISCAL POLICY SUSTAINABILITY, CASE OF GEORGIA
}

\author{
MAIA GRIGOLIA \\ PhD Student, \\ Ivane Javakhishvili Tbilisi State University, Georgia \\ m.grigolia@iset.ge
}

KEYWORDS: FISCAL POLICY SUSTAINABILITY, OUTPUT SUSTAINABILITY, FISCAL STABILITY INDICATOR, ECONOMIC GROWTH.

For citation: Grigolia, M. (2019). Output Volatility Impacts on Fiscal Policy Sustainability, Case of Georgia, Globalization And Business, №8, pp. 110-115. https://doi.org/10.35945/gb.2019.08.013

\section{SUMMARY}

The article discusses how fiscal stability affects macroeconomic sustainability and whether stability means strong economic growth in Georgia.The results of the analysis conducted in the article is supported by those numerous studies which indicate that fiscal stabilization reduces output volatility. Based on the existing analysis, we can say that fiscal policy can make a significant contribution to stabilizing output.

Fiscal Stability Indicator (FISCO) for Georgia has been calculated and cross-country analysis has been performed. It has been found that fiscal policy contributes more to stabilization of output in developed economies than in transitional markets and developing countries. The fiscal stabilization indicator for Georgia is 0.42 and is statistically significant, which indicates that one percentage point change in output causes 0.42 percentage point change in the total budget balance (as a share of GDP). The FISCO indicator is 0.41 for developed countries and 0.24 for transitional markets and emerging economies.
Based on the correlation analysis, it has been revealed that higher fiscal stability is associated with lower output volatility. However, here also, the difference between the groups of developed and transition and developing countries is significant: in developed countries- the relationship between fiscal stabilization and output fluctuation is stronger and sharply negative than in transition and developing economies.

More often, fiscal policy is used as a stabilization mechanism when the economy lags behind the desired pace of growth; And are less likely to resort to policy mechanisms when booming. Due to the proven importance of the fiscal stabilization in economic sustainability it can be concluded that the use of fiscal stabilization as a mechanism only in the "black days» can greatly worsen the sustainability of government debt, as governments appear to lack the advantage that they can reduce deficits and create fiscal buffers to better address future negative shocks in times of growth. 